\title{
ON CLOSED SUBSPACES OF OPERATOR RANGES
}

\author{
ROBIN HARTE AND GERRY SHANNON
}

(Communicated by Palle E. T. Jorgensen)

\begin{abstract}
Necessary and sufficient for the closure of a linear subspace to lie in the range of a bounded linear operator is a certain "bounded preimage property" for the operator.
\end{abstract}

If $T: X \rightarrow Y$ is a bounded linear operator between normed spaces then we shall, par abus de notation, also write [3]

$$
T: l_{\infty}(X) \rightarrow l_{\infty}(Y)
$$

for the operator induced between the corresponding spaces of bounded vectorvalued sequences

$$
l_{\infty}(X)=\left\{x \in X^{\mathbb{N}}: \sup _{n}\left\|x_{n}\right\|<\infty\right\} .
$$

1. Theorem. If $T \in \mathrm{BL}(X, Y)$ is a bounded linear operator between Banach spaces and if $M \subseteq Y$ is a linear subspace, then there is equivalence

$$
\operatorname{cl} M \subseteq T(X) \Leftrightarrow l_{\infty}(M) \subseteq T l_{\infty}(X) .
$$

Proof. We shall show forward implication for complete $X$ and backward implication for complete $Y$. Whether or not either space is complete, the right-hand side of (1.1) is equivalent to

$$
T_{M}^{\wedge}: T^{-1}(M) / T^{-1}(0) \rightarrow Y \text { bounded below. }
$$

Indeed if (1.2) holds then there is $k>0$ for which

$$
\operatorname{dist}\left(x, T^{-1}(0)\right) \leq k\|T x\| \text { for each } x \in T^{-1}(M),
$$

so that if $y \in l_{\infty}(M)$ is arbitrary then there is $x \in X^{\mathbb{N}}$ for which

$$
y=T x \quad \text { with } \operatorname{dist}\left(x_{n}, T^{-1}(0)\right) \leq k\left\|y_{n}\right\|,
$$

and then $z \in T^{-1}(0)^{\mathbb{N}}$ for which

$$
\|x-z\| \leq 2 \operatorname{dist}\left(x, T^{-1}(0)\right),
$$

giving

$$
y=T(x-z) \text { with } x-z \in l_{\infty}(X) .
$$

Received by the editors September 1, 1991.

1991 Mathematics Subject Classification. Primary 47A05; Secondary 47B07, 46B08.

Key words and phrases. Operator ranges, bounded sequences, Calkin property. 
Conversely if (1.2) fails then there is $x \in X^{\mathbb{N}}$ for which

$$
T x_{n} \in M, \quad\left\|T x_{n}\right\| \rightarrow 0, \quad \operatorname{dist}\left(x_{n}, T^{-1}(0)\right) \geq 1 .
$$

Now with

$$
x_{n}^{\prime}= \begin{cases}\left\|T x_{n}\right\|^{-1 / 2} x_{n} & \text { if } T x_{n} \neq 0, \\ n x_{n} & \text { if } T x_{n}=0,\end{cases}
$$

we have $\left\|T x_{n}^{\prime}\right\| \rightarrow 0$ and $\operatorname{dist}\left(x_{n}^{\prime}, T^{-1}(0)\right) \rightarrow \infty$ so that

$$
T x^{\prime} \in c_{0}(M) \subseteq l_{\infty}(M) \text { and } T x^{\prime} \notin T l_{\infty}(X) .
$$

If, in particular, the spaces $X$ and $Y$ are complete then condition (1.2) is also equivalent to the left-hand side of (1.1). To see this we need an auxiliary subspace

$$
M^{\sim}=T \operatorname{cl} T^{-1}(M)
$$

Evidently

$$
M \subseteq M^{\sim} \subseteq T(X) \cap \operatorname{cl} M,
$$

and hence, in particular,

$$
T_{M}^{\wedge} \text { bounded below } \Leftrightarrow T_{M^{\sim}}^{\wedge} \text { bounded below. }
$$

The operator $T_{M^{\sim}}^{\sim}$ is one-to-one, with range $M^{\sim}$, and if $X$ is complete defined on the complete space

$$
T^{-1}\left(M^{\sim}\right) / T^{-1}(0)=\operatorname{cl} T^{-1}(M) / T^{-1}(0),
$$

so that

$$
T_{M^{\sim}}^{\wedge} \text { bounded below } \Rightarrow M^{\sim}=\operatorname{cl} M^{\sim}
$$

since $M^{\sim}$ is complete. By (1.6) this gives

$$
M^{\sim}=\operatorname{cl} M,
$$

and hence also the left-hand side of (1.1) holds. Conversely if this happens then $\operatorname{cl} M$ is complete (if $Y$ is) and the open mapping theorem gives

$$
T_{\mathrm{cl} M}^{\wedge}: T^{-1}(\mathrm{cl} M) / T^{-1}(0) \rightarrow Y \text { bounded below, }
$$

and hence also (1.2).

The same argument gives the analogue of Theorem 1 in which the right-hand side of $(1.1)$ is replaced by the corresponding property for subsets

$$
\beta(M) \subseteq T \beta(X),
$$

where $\beta(X)$ denotes the bounded subsets of $X$; an easy consequence is that compact operators on complete spaces have the "Calkin property" $[4 ; 2$, Theorem III.1.12]

$$
\text { cl } M \subseteq T(X) \Rightarrow M \text { finite dimensional. }
$$

Notice that we have proved two versions of Theorem 1: we also have

$$
\operatorname{cl} M \subseteq T(X) \Leftrightarrow c_{0}(M) \subseteq T l_{\infty}(X) .
$$

In the particular case $M=T(X)$ Albrecht and Mehta [1, Lemma 2.1] have shown that also

$$
\operatorname{cl} M \subseteq T(X) \Leftrightarrow l_{\infty}(M) \subseteq T(X)+c_{0}(Y),
$$

which says that the image of $M$ in the "enlargement" of $Y$ [3, Definition 1.9.2] is included in the range of the enlargement of $T$. 


\section{REFERENCES}

1. E. Albrecht and R. D. Mehta, Some remarks on local spectral theory, J. Oper. Theory 2 (1984), 285-317.

2. S. Goldberg, Unbounded linear operators, McGraw Hill, New York, 1966.

3. R. E. Harte, Invertibility and singularity, Dekker, New York, 1988.

4. Ju. N. Vladimirskiĭ, Observations on Calkin operators, Siberian Math. J. 17 (1976), 715717.

Department of Pure Mathematics, Queen's University of Belfast, Belfast BT7 1NN, IRELAND

E-mail address: r.harte@v2.qub.ac.uk

Department of Mathematics, University of Ulster, Coleraine BT52 1SA, Ireland E-mail address: cdbql3@ucvax.ulster.ac.uk 